\title{
Terapia Ocupacional: una perspectiva histórica desde la Universidad Nacional de Colombia (1966-1989) ${ }^{1}$
}

\author{
Occupational Therapy: a historical perspective \\ from Universidad Nacional de Colombia (1966-1989)
}

\author{
Laura Rodríguez Mendoza² \\ Nathalia Camargo Carrero ${ }^{3}$ \\ Xiomara Escobar Jiménez ${ }^{4}$
}

Recibido: 25 de octubre de 2016 • Enviado para modificación: 11 de enero de 2017 • Aceptado: 24 de febrero de 2017. Rodríguez, L., Camargo, N., \& Escobar, X. (2016). Terapia Ocupacional: una perspectiva histórica desde la Universidad Nacional de Colombia. Revista Ocupación Humana, 16 (2), 26-45.

Resumen: Este trabajo constituye uno de los primeros aportes al macroproyecto Historias de las Terapias Ocupacionales en Colombia. La investigación describe los hitos y sucesos que antecedieron, dieron origen y evolución al primer programa de formación en Terapia Ocupacional en el país, ofrecido por la Universidad Nacional de Colombia. Se empleó la metodología de investigación histórica a partir del reconocimiento de tres categorías: sujetos, discursos e instituciones; las cuales convergen en los contextos sociales, políticos, económicos y culturales de las décadas de los sesenta, setenta y ochenta, y en los escenarios internacional, nacional, local (Bogotá), la Universidad Nacional y la carrera de Terapia Ocupacional. Las fuentes consultadas incluyen documentos y relatos de protagonistas del programa en el periodo estudiado. Con la información obtenida se consolidan tres líneas de tiempo, una por cada década, en las que se recuperan los planes y reformas curriculares de la formación técnica, transformada en licenciatura a partir de 1976 y configurada como profesional a partir del Decreto Ley 80 de 1980. Se invita a la realización de investigaciones en clave histórica como una posibilidad de empoderamiento profesional que aporta a la comprensión del devenir de la Terapia Ocupacional.

Palabras clave: Historia, Terapia Ocupacional, investigación histórica, memoria colectiva.

Abstract: This work constitutes one of the first contributions to the macro project: Stories of occupational therapies in Colombia. The research describes the milestones and events

\footnotetext{
1 Artículo derivado de la investigación Terapia Ocupacional: una perspectiva histórica desde la Universidad Nacional de Colombia (1966-1989), realizada por las autoras como trabajo de grado para optar al título de terapeutas ocupacionales de la Universidad Nacional de Colombia y dirigida por la docente Aleida Fernández Moreno. Trabajo ganador del segundo puesto del Premio de Investigación Alicia Trujillo Rojas, decima versión (2016), en la categoría estudiantes.

${ }^{2}$ Terapeuta Ocupacional. Estudiante Maestría en Neurociencias, Universidad Nacional de Colombia. Laboratorio desarrollo, juego, aprendizaje y lúdica, Departamento de la Ocupación Humana, Universidad Nacional de Colombia. Grupo de Investigación Historias de las Terapias Ocupacionales en Colombia - HiTOs. lvrodriguezm@unal.edu.co

${ }^{3}$ Terapeuta Ocupacional. Unidad Terapéutica Integral BITA. Grupo de Investigación Historias de las Terapias Ocupacionales en Colombia - HiTOs.nnaacamargoca@unal.edu.co

${ }^{4}$ Estudiante de Terapia Ocupacional, Universidad Nacional de Colombia.xcescobarj@unal.edu.co
} 
that preceded, and gave origin and evolution to the first training program in Occupational Therapy in the country, offered by the Universidad Nacional de Colombia. The methodology of historical research was used, from the recognition of three categories: subjects, discourses and institutions. These converge in the social, political, economic and cultural contexts of the 60s, 70s and 80s and in several scenarios: international, national, local (Bogotá), the Universidad Nacional and the occupational therapy program. The sources consulted include documents and stories of central characters of the program in the period studied. With the information gathered, three timelines were obtained, one for each decade. The lines retrieve plans and curricular reforms to technical training, transformed into a Bachelor's degree from 1976 and configured as a professional degree in Law 80 of 1980. We invite to the conduction of research in historical terms, as a possibility for professional empowerment that contributes to the understanding of the evolution of occupational therapy.

Key words: History, occupational therapy, historical research, collective memory.

\section{Introducción}

La historia continúa, es el correlato indispensable de la función fundadora del sujeto: la garantía de que todo cuanto le ha escapado podrá serle devuelto;

la certidumbre de que el tiempo no dispersará nada sin restituirlo en una unidad recompuesta; la promesa de que el sujeto podrá un día (bajo la forma de la conciencia histórica), apropiarse nuevamente todas esas cosas mantenidas

lejanas por la diferencia, restaurará su poderío sobre ellas y en ellas encontrará lo que se puede muy bien llamar su morada.

Foucault, 1979, p. 21.

Esta investigación estuvo dirigida a aportar desde la Universidad Nacional de Colombia a la reconstrucción histórica de la Terapia Ocupacional en el país, como respuesta al Ilamado que con ese fin realizó desde 2014 el Grupo de Investigación Historias de las Terapias Ocupacionales- HiTOs. Como tal, se centró en la descripción del devenir histórico del primer programa académico de Terapia Ocupacional en Colombia en las décadas de los sesenta, setenta y ochenta del siglo XX. Se busca con él que las y los terapeutas ocupacionales, profesionales y en formación, así como las nuevas generaciones, puedan conocer registros primarios y hacer una lectura detallada de los antecedentes del surgimiento de la formación en Terapia Ocupacional en la Universidad Nacional de Colombia. Se toma como referente uno de los fundamentos del Grupo HiTOs: "reconocer el pasado para comprender el presente y visualizar el futuro" (Fernández, Cruz, Duarte, \& García, 2015).

Para ello, la investigación tuvo en cuenta la perspectiva para la reconstrucción histórica planteada por el sociólogo y filósofo Michael Foucault, quien sugería tener en cuenta tres categorías: en primer lugar los Sujetos, entendidos como personas bajo sus distintas nominaciones históricas, con el fin de vislumbrar la evolución terminológica (terapeutas ocupacionales, técnicas, licenciadas); las instituciones, lugares en los que se desarrolló la profesión o en los que se dieron los sucesos, y donde emergen los discursos, 
entendidos como una "enunciación individual o correlativa" que permite la aproximación a los hechos y a las transformaciones generadas (Foucault, 1979).

A su vez, se identificó que todo hecho era determinado por un contexto configurado cronológica y sistémicamente, por lo que de forma breve y general se sustentan los contextos político, económico, social y cultural. El contexto político se refiere a sucesos en un determinado espacio de deliberación pública y de poder (Mouffe, 1999); el económico, a las formas de utilización, administración y distribución de los recursos limitados para el consumo y la satisfacción de las necesidades de los miembros de la sociedad (Capó, s.f.); el social engloba a los grupos que conforman distintas organizaciones que construyen lo cultural, influyendo y trascendiendo en costumbres y acciones que definen la identidad de las sociedades en un tiempo y espacio (Reyes, 2011). Tales contextos se entienden en las esferas macro (internacional y Latinoamérica), meso (nacional y Universidad Nacional) y micro (carrera de Terapia Ocupacional).

De esta manera, como lo sugiere (Foucault, 1979), se transformaron "Ios documentos, en monumentos" de tal forma que se describiera de forma comprensiva y veraz la trayectoria histórica de sucesos significativos en la Terapia Ocupacional de la Universidad Nacional en las tres décadas abarcadas. Estos son: el proceso de transformación de la titulación de terapeutas ocupacionales, inicialmente como técnicos, luego licenciados y finalmente profesionales, y la configuración de las prácticas o ejercicio profesional, entre otros resultados emergentes.

Finalmente, con esta investigación se logró aportar a la invitación que hiciera la profesora emérita Alicia Trujillo (2002), a propósito de la importancia de estudiar la historia de la Terapia Ocupacional: "En Colombia, las fuentes que estudian las raíces sociales de la Terapia Ocupacional son casi inexistentes (...). Una profesión como la Terapia Ocupacional no puede sustraerse de reconstruir y entender la crucial influencia que las condiciones culturales y eventos y coyunturas políticas, económicas, educativas, de salud y de trabajo han ejercido en su aparición y configuración"(p.472).

\section{Metodología}

La metodología de investigación histórica, al ser utilizada por primera vez dentro de un grupo cuyas integrantes son terapeutas ocupacionales, no historiadoras, se convirtió en un reto. En este trabajo se adelantaron cuatro etapas: contextualización; definición de categorías y archivos; lectura y categorización de información, y escritura descriptiva alrededor de los hallazgos. Estas etapas se fueron configurando de la mano del Seminario Permanente, estrategia que ha hecho parte del desarrollo del macroproyecto Historias de las Terapias Ocupacionales en Colombia.

La etapa de contextualización tuvo como fundamentos los aprendizajes y acuerdos de trabajo de las dos primeras sesiones presenciales del Semina- 
rio, realizadas en Bogotá, en julio de 2014, y en Cali, en octubre del mismo año (HiTOs, 2014). En ellas se abarcaron: lo que se comprende por historia, cómo comprenderla, cómo justificar el estudio histórico y estructurarlo, la construcción del archivo. Desde allí se establecieron las primeras categorías de análisis y objetivos.

Más adelante, en la sesión realizada en la ciudad de Pasto, emerge la etapa de definición de categorías y archivos. Con el apoyo de expertos se analizaron algunas categorías, se definieron los sujetos, discursos, instituciones y contextos que darían sustento a la clasificación de la información encontrada en términos de acontecimientos en esferas sistémicas. Allí se determinó que los discursos no solo podían constituirse a partir de la lectura de documentos escritos, también mediante recursos narrativos y utilizando dispositivos para activar y aprovechar la memoria viva y colectiva de las precursoras de la profesión.
Con base en este aprendizaje se convocó a las profesoras pensionadas y de larga trayectoria en el programa de la Universidad Nacional a un Té de homenaje, el cual se realizó el 21 de abril de 2015 y contó con las narrativas de Alicia Trujillo Rojas, Laura Álvarez de Bello, Emma Grimaldo, Ana María Gómez y María Victoria Zapata (Figura 1), logrando recolectar información desde sus experiencias y trayectorias. De otro lado, en la Línea de Profundización de Estudios en Discapacidad se realizaron conversatorios individuales con otras profesoras pensionadas y egresadas de las primeras cohortes.

Por otra parte, el Archivo Histórico y Central, y la Biblioteca Gabriel García Márquez de la Universidad Nacional de Colombia, sumados a las bibliotecas personales de algunas docentes que acompañaron el proyecto, se constituyeron en fuentes de información documental.

Figura 1. Profesoras y autoras durante el Té de Homenaje, abril de 2015.

Parte superior, de izquierda a derecha: Alicia Trujillo Rojas, Laura Álvarez de Bello, María Victoria Zapata, Emma Grimaldo y Ana María Gómez. Parte inferior, de izquierda a derecha: Xiomara Escobar, Nathalia Camargo y Laura Rodríguez.

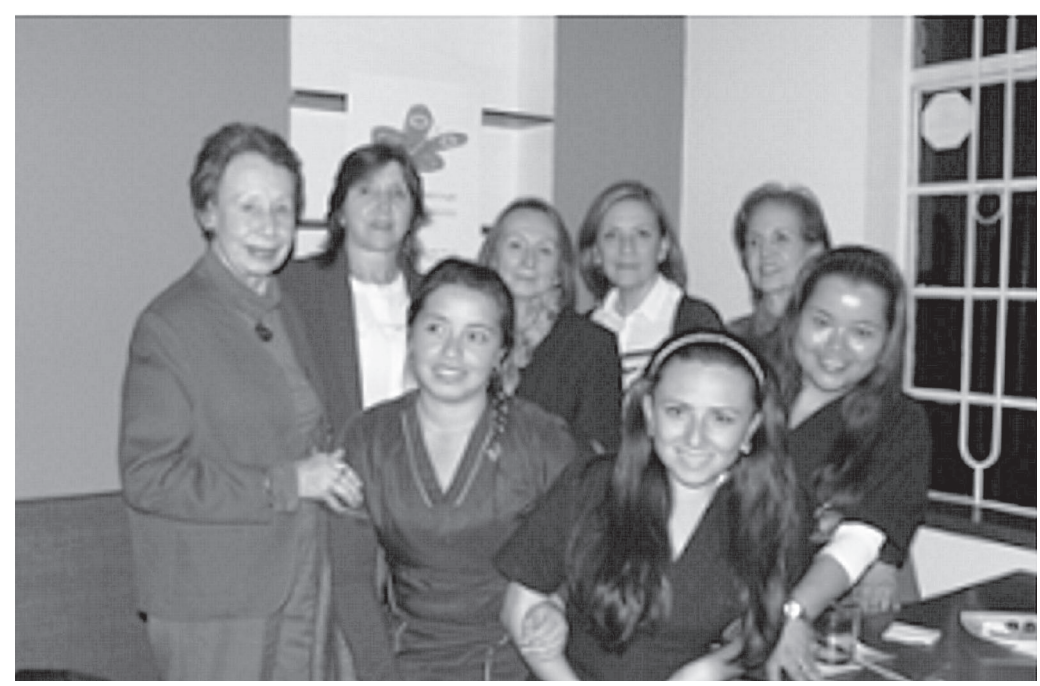


Posteriormente se realizó la lectura y categorización de información, que recogió las reflexiones del seminario llevado a cabo en la Escuela Colombiana de Rehabilitación en junio de 2015 (HiTOs, 2015). En esta etapa se estableció una matriz de síntesis de la información recolectada, la cual facilitó su clasificación en las categorías y contextos definidos. Luego se hizo una lectura transversal de esas categorías y contextos década por década, y se trazó una matriz que más adelante se convirtió en líneas de tiempo. Esto permitió configurar cronológicamente los hechos históricos de forma legible. Con este ejercicio de análisis se evidenciaron además hechos cuya importancia se reiteraba; uno de ellos, el proceso de transformación del programa curricular y del título de formación obtenido. La triangulación de fuentes (estudiante de la época, docente y normativa) permitió identificar hitos históricos en ese proceso.

Finalmente, se realizó la escritura descriptiva alrededor de los hallazgos, para lo cual se asistió a dos talleres de escritura coordinados por la Terapeuta Ocupacional Solángel García, integrante del grupo HiTOs. De esta forma fue posible realizar una descripción que pasara del esquema a la narración, de forma que la historia contada lograra ser legible.

\section{Resultados}

\section{Antes de la creación del programa de Terapia Ocupacional, el ambiente de las primeras décadas del Siglo XX}

A nivel internacional iniciaron las luchas políticas entre capitalismo y comunismo como la Guerra de Corea (1946 - 1950), la Guerra Fría (1953 1962) y, en Latinoamérica, el periodo de transición de la Revolución Cubana en 1953, que finalizó con la posesión de Fidel Castro como presidente de Cuba en 1959 (Harina, 2010).

Mientras tanto, en Bogotá tuvo lugar un periodo de violencia que resultó del asesinato del caudillo liberal Jorge Eliécer Gaitán en 1948, el cual desencadenó El Bogotazo. Para esa época Colombia se dividía ideológicamente en dos partidos políticos, liberal y conservador, cuyos debates por el poder dieron pie a la conformación del Frente Nacional, "sinónimo de movilización social, sindicalismo y movimientos estudiantiles" (Gutiérrez, 2015). Atendiendo a las necesidades políticas emergentes luego del Bogotazo, en 1954 las mujeres obtuvieron el derecho al voto; sin embargo, solo hasta 1957 pudieron ejercerlo por primera vez. Con el sufragio femenino se consolidaban las luchas originadas en los años treinta (Cohen, 2001).

Hacia finales de la década de los cincuenta el total de estudiantes de la Universidad Nacional de Colombia, mayoritariamente hombres, representaban el $50 \%$ de aquellos que ingresaban a la educación superior en el país tras cumplir la exigencia de culminar sus estudios como bachilleres. Las mujeres entre tanto eran tenidas en cuenta en la "universidad femenina" 5 para ser formadas según sus características

\footnotetext{
${ }^{5}$ Para Cohen (2001), la universidad femenina representó el intento de algunos grupos de mujeres por ocupar espacios de poder, controlando estas instituciones a través de alternativas educativas prácticas que permitieran
} 
y sin que "compitieran con los varones por los mismos puestos de trabajo" (López, 2002, p. 71). Las que accedían a la universidad superaban los prejuicios al ser minoría, salvo quienes ingresaban a estudiar Enfermería, dado que ya contaban, como gremio, con un estatus profesional (Gómez, et al., 2011).

\section{Década de los sesenta: el augurio de grandes cambios}

Por esta época, a nivel internacional, la instalación soviética de plataformas lanza misiles en territorio cubano representó para la Casa Blanca una amenaza nuclear sin precedentes, de tal magnitud que el mundo se arriesgaba a comenzar una Tercera Guerra Mundial. Fue entonces cuando Cuba comenzó a "exportar" la revolución comunista al resto de los países latinoamericanos con el apoyo económico y político de la Unión Soviética, lo que significaba una agresión al sistema democrático capitalista pregonado, "difundido y hasta impuesto por los Estados Unidos de América en el resto de los países latinoamericanos" (Mengo, 2004, p. 42).

Lo anterior influyó en una serie de transformaciones culturales que promovieron el reconocimiento de la diversidad de pensamiento, especialmente político. Esto se manifestaba en un tipo de escritura tensionante que dinamizó la expresión de las nuevas concepciones entre quienes escribían y sus relaciones con el Estado. Las corrientes feministas lograron incremen- tar el número de mujeres vinculadas a programas de educación universitaria (UNESCO, 1998), y la memoria cultural que compilaron los intelectuales con sus ideas o acciones ayudó a fomentar la construcción de una utopía revolucionaria que promovió la modernización universitaria y la crítica hacia el modelo de educación superior norteamericano. Todo ello tras la ruptura de las relaciones políticas, económicas, sociales y culturales de Cuba con los Estados Unidos, que dio su nombre a la Revolución Cubana (Urrego, 2002).

Finalizando la década, en 1969, el hombre llegó a la luna ocho años después de viajar por primera vez al espacio. Este hecho representó el cumplimiento del sueño del presidente de Estados Unidos John F. Kennedy, asesinado en 1963, quien lo visualizaba como una forma de superar los avances científicos y tecnológicos de la Unión Soviética (Dunbar, 2008).

Kennedy visitó Colombia en 1961, hecho que consolidaba la Alianza para el Progreso, un programa de ayuda externa a América Latina propuesto por Estados Unidos con el fin de crear condiciones para el desarrollo y la estabilidad política y económica de la región, y con el cual se habría de financiar mayoritariamente la Reforma Patiño en la Universidad Nacional de Colombia (Carrillo, 2014).

Posteriormente, en 1966, se conformaron en Colombia los grupos guerrilleros Ejército de Liberación Nacional

a las mujeres hacer compatible su vida familiar y profesional. Fue una manera de influir en la transformación de los valores imperantes en la sociedad, convencidas de que ellas mismas debían crear nuevos modelos. 
(ELN) y Fuerzas Armadas Revolucionarias de Colombia (FARC), ello como fruto de la exclusión social y política, la distribución desigual de las tierras y la injusticia social vividas hasta ese momento en el país (Mengo, 2004).

Por otra parte, la Universidad Nacional de Colombia empezó a reconocerse como una institución pública, autónoma y con identidad corporativa legal por medio del proyecto de reforma universitaria establecido por el médico y rector de la Universidad José Félix Patiño. Dicha reforma, aprobada por el Consejo Superior Universitario a través del Acuerdo 059 del 25 de marzo de 1965, constituyó la entonces llamada ciudad universitaria (Gutiérrez, 2015), estableció la división académica de la universidad y definió sus funciones y organización general luego de la crisis de infraestructura y bienestar en la que se encontraba (Mengo, 2004).

El 1 de septiembre de 1959 la Facultad de Medicina de la Universidad había creado el Servicio de Medicina Física y Rehabilitación, una unidad dependiente de la sección de Medicina Interna del Hospital San Juan de Dios que efectuaba actividades administrativas, docentes y asistenciales, las cuales dieron origen en 1960 a la formación posgraduada de médicos especialistas en Medicina Física y Rehabilitación en la Universidad (Pardo, 1972).

Posteriormente las directivas de la Facultad de Medicina detectaron una amplia demanda de servicios en la sec- ción de rehabilitación, derivada de la situación de violencia que atravesaba el país, por lo que vieron la necesidad de constituir equipos completos de salud en rehabilitación (Pardo, 1972). En consecuencia, desde el Hospital San Juan de Dios se solicitó a la Facultad cubrir estas necesidades por medio de la formación en el área. Así, mediante el Acuerdo 04 del 20 de enero de 1966 (Consejo Superior Universitario, 1966), el Consejo Superior Universitario autorizó a la Facultad de Medicina para que en la "Sección de Rehabilitación" iniciaran labores tres carreras técnicas que vendrían a llenar el vacío existente en el campo de las ciencias de la salud: Terapia Física, Terapia de Lenguaje y Terapia Ocupacional (Pardo, s.f.). Inicialmente las admisiones serían semestrales.

Los tres programas creados tenían una duración de seis semestres y compartían en el primer año asignaturas como Anatomía, Física, Psicología General, Sociología, Orientación, Primeros Auxilios, Fisiología Neuromuscular, Anatomía Funcional, Patología General y Neuromuscular, Psicología Médica, Kinesiología e Inglés; posteriormente recibían clases propias de cada área. Al terminar se recibía el título de técnico (Pardo, s.f). En 17 de diciembre de 1968 se presentó el primer Acta de Grado Colectivo para Técnicas en Terapia Ocupacional de la Universidad Nacional de Colombia, con un total de nueve graduandas ${ }^{6}$.

Para el caso de Terapia Ocupacional, inicialmente la dirección del programa estuvo a cargo del director del Depar-

${ }^{6}$ El Acta fue recuperada y transcrita, está disponible en el informe final del trabajo de grado. 
tamento de Rehabilitación, Jorge Pardo Ruiz, y las terapeutas ocupacionales Patricia Ann Lang de Pardo, de procedencia Norteamericana y graduada de la Western Michigan University (Registro de personal docente, s.f.), y Alicia Trujillo Rojas, colombiana con formación en la New York University (Trujillo, 2002).

\section{Instituciones y prácticas en los sesenta}

Según lo establecido en el Acuerdo 04 de 1966 del Consejo Superior Universitario (Consejo Superior Universitario,1966), a partir del segundo año de formación el mayor número de horas a la semana se dedicaba a las Prácticas Clínicas, Férulas y Adaptación de Equipos, Artes Manuales y Artesanía. En el tercer año se realizaban prácticas clínicas en Pediatría, Psiquiatría y Rehabilitación, que para la época implicaban la asistencia al Hospital San Juan de Dios, principalmente, así como a otras instituciones. Así lo confirma la terapeuta ocupacional, Emma Grimaldo, docente pensionada de la Universidad Nacional de Colombia:

...Aquí [refiriéndose a la Universidad] recibíamos Kinesiología en un salón de Odontología y nos la daba el Dr. Pardo, el Dr. Rangel, el Dr. Avelin Rustedes, el Dr. Vega, todos esos doctores nos daban Kinesiología, y entonces Morfo aquí (...) eran todos los médicos...claro, el profesor Rubiano, todos los médicos (...). Luego, después eran como tres bloques, tres áreas, que era en ese entonces Incapacidades Físicas en Niños, que era Incapacidades Físicas en Adultos, que era en San Juan de Dios (...) Bueno... Patricia de Pardo, excelente profesora, con nosotras era (...) exigente, disciplinada, ella manejaba San Juan de Dios, nos dictaba en la teoría de Incapacidades Físicas, nos hacía la práctica ahí en el Servicio de San Juan de Dios, entonces ella fue una de mis profesoras. Después en la parte de niños mi profesora fue Margarita Rojas, que era de la primera promoción, (...) Salud Mental en la clínica Santo Tomás, y entonces allá cuando a mí me tocó Salud mental mi profesora fue Alicia (...). En la Unidad del San Juan íbamos era a una clase de Psiquiatría... sólamente en San Juan con doña Patricia, en Santo Tomás con Alicia, y en Propace ${ }^{7}$ con Margarita Rojas... también recibí clase de Conferencias Clínicas con los profesores que nos daban Kinesiología, también nos dictaban otra vez conferencias clínicas de todas las patologías, todo lo de patología nos lo dictaban esos doctores (...) por la tarde veníamos aquí a la universidad y nos dictaban Cerámica (...) y Férulas también las hicimos allá en San Juan de Dios en un salón grande que nos tocó limpiar y arreglar... (E. Grimaldo, comunicación personal, 15 de marzo de 2015).

Respaldando lo mencionado por la profesora Grimaldo, el Hospital San Juan de Dios es identificado por las docentes como la institución más importante para el programa de Terapia Ocupacional de la Universidad Nacional de Colombia, dado que es allí donde surgió. Sobre este espacio la profesora Nohora Díaz refiere:

...teníamos cuatro espacios, al principio estábamos en la Sección de Hos-

\footnotetext{
${ }^{7}$ Asociación Colombiana Pro Niño con Parálisis Cerebral- PROPACE.
} 
pitalización y Salud Mental, también entrabamos a Cuidados Intensivos. Luego cuando las personas salían de estar hospitalizadas ingresaban a Rehabilitación, teníamos talleres de artes, con hiladoras, cerámicas y en fin... Dibujé caras felices porque siempre las personas entraban y salían muy felices del servicio de Terapia Ocupacional, teníamos incluso una cafetería... (N. Díaz, comunicación personal, 11 de marzo de 2015).

En 1967 se instauró en el Hospital de La Misericordia otro servicio adjunto de rehabilitación para la atención de niños con todo tipo de "incapacidad". Un año después se creó allí "la escuelita de educación especial", donde se atendían niños con "incapacidades físicas" y trastornos severos, pensando en aquellos que no podían asistir a la escuela regular por su condición de hospitalización (Pardo, 1972). En la Tabla 1 se relacionan las instituciones en la que se desarrollaron prácticas del programa de Terapia Ocupacional en la década del sesenta.

Tabla 1. Instituciones donde desarrolló prácticas el programa de Terapia Ocupacional en la década de 1960.

\begin{tabular}{|l|l|l|l|}
\hline $\begin{array}{c}\text { Año } \\
\text { de inicio }\end{array}$ & \multicolumn{1}{|c|}{ Institución } & \multicolumn{1}{|c|}{ Práctica académica } & \multicolumn{1}{|c|}{ Docente a cargo } \\
\hline $\mathbf{1 9 6 6}$ & Hospital San Juan de Dios. & $\begin{array}{l}\text { Disfunciones Físicas niños. } \\
\text { Disfunciones Físicas } \\
\text { adultos. }\end{array}$ & $\begin{array}{l}\text { Jorge Pardo } \\
\text { Alicia Trujillo } \\
\text { Patricia de Pardo } \\
\text { Margarita Rojas }\end{array}$ \\
\hline $\mathbf{1 9 6 7}$ & $\begin{array}{l}\text { Clínica Santo Tomás } \\
\text { Hospital de la Misericordia. }\end{array}$ & $\begin{array}{l}\text { Salud Mental } \\
\text { Disfunciones Físicas niños. }\end{array}$ & $\begin{array}{l}\text { Alicia Trujillo } \\
\text { Maria Helena Rubio }\end{array}$ \\
\hline $\mathbf{1 9 6 8}$ & $\begin{array}{l}\text { Asociación Colombiana Pro } \\
\text { niño con Parálisis Cerebral - } \\
\text { Propace. }\end{array}$ & Disfunciones físicas niños. & $\begin{array}{l}\text { Margarita Rojas } \\
\text { Emma Grimaldo }\end{array}$ \\
\hline
\end{tabular}

Fuente: Elaboración propia a partir de los registros de archivo.

Década de los setenta: la licenciatura, implementación de cambios de inicio a fin

Los setentas constituyeron una década de transformaciones. A nivel Internacional, "la Guerra Fría muestra una larga y abierta rivalidad que enfrentó a Estados Unidos y la Unión Soviética y sus respectivos aliados tras la Segunda Guerra Mundial. Este conflicto fue la clave de las relaciones internacionales mundiales durante casi medio siglo y se libró en los frentes políticos y económicos" (Hobsbawn, 2000).

En Latinoamérica ocurrieron crisis políticas trascendentales. En Chile, el golpe de Estado contra el presidente Allende en 1973 dio paso a la dictadura militar del General Augusto Pinochet, lo que significó para miles de chilenos el comienzo de una época de exilios, desapariciones y torturas (Ministerio de Educación, Gobierno de Chile, 2014). Situaciones similares vi- 
vieron los argentinos bajo la dictadura de Videla, que inició en 1976, y los uruguayos, desde 1973, con Juan María Bordaberry (Anderson, 1988).

A inicios de los setenta nació en Colombia el grupo guerrillero Movimiento 19 de Abril - M-19, el cual protagonizó en ese decenio sucesos como el robo de la espada de Bolívar, la toma de la Embajada de República Dominicana y el robo de armas del Cantón Norte (Jaimes, 2012). Durante la presidencia de Alfonso López Michelsen se presentó uno de los más fuertes movimientos de inconformismo en el país durante el siglo XX, el Paro Cívico Nacional de 1977 convocado por las centrales sindicales del país, cuyas motivaciones incluían la situación de carestía que campeaba y las altas cifras de desempleo (Tribuna Roja, 1977).

De otro lado, desde la rectoría de Mario La Torre Rueda, en los años setenta se impulsaron los primeros programas de educación posgraduada en la Universidad Nacional de Colombia. Entre 1967 y 1973 fueron creadas las maestrías en Administración y en Educación, primeras en el país y en América Latina. Adicionalmente, la Universidad otorgaba becas a quienes se graduaban en condiciones de excelencia académica; así lo relata la profesora Emma Grimaldo para el caso de Terapia Ocupacional:

Quien obtenía el título de técnica en Terapia Ocupacional y lograra grado de honor, la Universidad le daba una beca para ir a estudiar al extranjero, a Nueva York. Yo fui al Norwood Memorial Hospital y el Dr. Pardo también me envió una nota a Howard Ruks en Nueva York, y él me recibió y me mostró todo lo que el manejaba en rehabilitación... increíble. (E.Grimaldo, comunicación personal, 15 de marzo de 2015).

Por otra parte en la Facultad de Medicina, durante la decanatura de Carlos Cuervo en 1972, la profesora Alicia Trujillo buscaba atender las necesidades de actualización y continuidad en la formación de quienes egresaban del programa de Terapia Ocupacional (A.Trujillo, comunicación personal, 21 de abril de 2015). Al respecto, la profesora Laura Álvarez de Bello relata:

Las terapeutas ocupacionales para el año 1972 empezaron a realizar un curso en el Centro de Rehabilitación Profesional para inválidos, que quedaba en la Caracas con 24 y estaba dirigido por Hillary Schlesinger, que era una delegada de la OIT. (L. Álvarez, comunicación personal, 21 de abril de 2015).

Más allá de estos esfuerzos, el interés de docentes, estudiantes y egresadas por profesionalizar la carrera, que se materializó en el logro de la licenciatura en 1976, constituye para la historia del programa de Terapia Ocupacional de la Universidad Nacional de Colombia el hito más importante de la década de los setentas.

Concentradas en ello y con la participación activa de toda la comunidad académica del programa, incluyendo un importante liderazgo estudiantil, en 1973 se emprendieron en la Universidad Nacional y en el Hospital San Juan de Dios "grandes luchas", como sus protagonistas las denominan, para impulsar la formación profesional. Para corroborar esta información se 
realiza triangulación de tres discursos importantes, el primero legislativo (Acuerdo 15 de 1976 del Consejo Superior Universitario) y los otros dos COrrespondientes a las narraciones de la profesora Alicia Trujillo y la Terapeuta Ocupacional Aida Navas, quien para entonces era estudiante.

De este proceso se derivaron cambios estructurales en el plan curricular del programa, en donde se destacan la introducción de la investigación y la implementación de las estrategias de nivelación y actualización necesarias para que quienes se habían graduado como técnicas pasaran a ser licenciadas.

\section{Primera reforma curricular del programa}

La primera revisión curricular del programa se consolidó en el Acuerdo 15 de 1976 del Consejo Superior Universitario, el cual dio paso al Acuerdo 0016 de 1977 del Consejo Superior Universitario (Acta 7 del 15 de enero), a través del cual se aprobó el plan de estudios para la Licenciatura de Terapia Ocupacional. En esta reforma se implementaron bases teóricas, metodológicas y aplicadas a la investigación, exigiendo la realización de un registro monográfico como requisito de grado (Peñas, 2006).

Adicionalmente, quienes para entonces tenían el título de técnicas (algunas de las cuales ejercían ya como docentes del programa) debían presentar y aprobar un examen para acceder al título de licenciadas: "...tuvimos que preparar un curso para las demás y así podían obtener el título de licenciado"
(L. Álvarez, comunicación personal, 21 de abril de 2015). Estas personas recibieron su segundo grado el 22 de abril de 1977.

De otra parte, Trujillo, Torres, Méndez y Carrizosa (2011) refieren que "en este momento las profesoras de Terapia Ocupacional comenzaron a ocupar cargos académico administrativos los cuales, a través de su gestión, favorecieron roles decisivos en el rumbo de sus programas"(p. 44). Esto se relaciona con otro hito en la historia del programa de Terapia Ocupacional de la Universidad Nacional de Colombia, la creación en 1978 del cargo de director académico para las carreras de Terapia del Lenguaje, Física y Ocupacional.

El Consejo Superior Universitario había reglamentado, a través del Acuerdo 83 del 20 de septiembre de 1977, los cargos y funciones de los directores académicos y los comités asesores de carrera; en consecuencia, en 1978, mediante el Acuerdo 12 del 21 de febrero del Consejo Superior Universitario "se provee una dirección independiente para las licenciaturas de terapias". En este último Acuerdo se consideró además el aumento de cupos de las "carreras de terapia", las cuales hasta el momento eran dirigidas solo por el director del Departamento de Rehabilitación, Dr. Jorge Pardo Ruiz, quien paralelamente dedicaba sus esfuerzos al pregrado de Medicina (cuyos cupos también aumentaban) y al programa de residencia clínica en la misma disciplina. De esta manera se autorizó "al Consejo Directivo de la Facultad de Medicina para proceder a nombrar un director académico de 
las carreras de Terapia del Lenguaje, Física y Ocupacional", y a "separar las funciones de director de departamento y de director académico de carre$\mathrm{ra}^{\prime \prime}$ (Consejo Superior Universitario, 1978).

\section{Instituciones y prácticas de los setenta}

Paralelamente, la academia siguió fortaleciendo sus espacios de incidencia en atención a las necesidades de la población. Para 1973 la profesora Laura Álvarez de Bello continuaba los programas y acciones para niños y niñas desescolarizados y con necesidades educativas especiales en el Hospital de la Misericordia, práctica que más adelante se consolidó en el área educativa:

Yo empecé en La Misericordia haciendo la práctica de niños, pero La Misericordia tenía una escuelita, que era la escuela de sordos en 1973, entonces en 1974 hablando con muchos niños que no tenían escolaridad logramos que el Dr. Gustavo Vega aceptara incluir niños con déficit cognitivo, eran los retardados mentales en ese momento. (L. Álvarez, comunicación personal, 21 de abril de 2015).

Al mismo tiempo se ampliaron los servicios en el Hospital San Juan de Dios, en donde se prestaba atención de Terapia Ocupacional en el área pre vocacional y de orientación laboral; también se empezaban a realizar análisis de puestos de trabajo desde la Universidad (Laserna, 1995). En 1973, luego de que desaparece el Consejo Nacional de Rehabilitación, se crea el Instituto Colombiano de Seguro Social, como lo explica la Te- rapeuta Ocupacional Margarita González:

Vino el Seguro Social que quería formar su primer Centro de Rehabilitación Profesional, y ahí estaban Carmen Helena Vergara y Hirally Schlesinger, inglesa, (...) ella era experta, se llamaba experta consultora de la Organización internacional del Trabajo (OIT), y había formado el primer Centro de Rehabilitación Profesional en Argentina con Antonio Lacar, quien fue el director del GLARP en la última etapa en Colombia, prácticamente. (M. González, comunicación personal, 20 de marzo de 2015).

Por otra parte, Terapia Ocupacional apoyaba con la profesora Emma Grimaldo el Programa Madre Canguro, concebido en 1978 en el Hospital Materno Infantil para atender las necesidades de bebés prematuros cuyo delicado manejo requería capacitar a las madres, familiares y al personal hospitalario (Instituto Materno Infantil, 2003).

... había mucho hacinamiento, mucha crisis de que no tenían como manejar a esos niños... entonces empezaron ahí en el Materno, después fue cuando con UNICEF consiguieron plata para hacer la casita... empecé yo en el Materno con estudiantes, y entonces yo hacía eso con el Dr. Hector Martínez, él era el encargado de los prematuros, entonces él los miraba y yo en el mismo consultorio, en la casita, evaluaba. (E. Grimaldo, comunicación personal, 15 de marzo de 2015).

Adicionalmente, las prácticas de rehabilitación en disfunciones físicas en niños y adultos en el Hospital San Juan del Dios se mantuvieron en esta 
década, y se dio por finalizado el convenio con la Clínica Santo Tomás en 1974 (Trujillo, 1981). En la Tabla 2 se resumen las instituciones en las que se desarrollaron prácticas de Terapia Ocupacional durante los setenta.

Tabla 2. Instituciones donde se inician prácticas del programa de Terapia Ocupacional durante la década de 1970.

\begin{tabular}{|c|c|c|c|}
\hline $\begin{array}{c}\text { Año } \\
\text { de inicio }\end{array}$ & Institución & Áreas & Docente a cargo \\
\hline 1972 & Hospital de la Misericordia & $\begin{array}{l}\text { Pabellón de Quemados. } \\
\text { Centro de Educación Espe- } \\
\text { cial - "La Escuelita". } \\
\text { Trastornos del Aprendizaje. }\end{array}$ & $\begin{array}{l}\text { Lucy Carrizosa } \\
\text { Laura Álvarez de Bello } \\
\text { María Helena Rubio }\end{array}$ \\
\hline 1973 & Instituto Materno Infantil & Neonatos & Emma Grimaldo \\
\hline 1974 & Hospital San Juan de Dios & Salud Mental & Alicia Trujillo \\
\hline 1978 & Hospital Julio Manrique - Sibaté & Salud Mental & \\
\hline
\end{tabular}

Fuente: Elaboración propia a partir de los registros de archivo.

Década de los ochenta: se consolida la profesionalización

Entre los hechos relevantes de este decenio a nivel Internacional, los países asiáticos experimentaron un rápido desarrollo industrial que aún no se detiene; aumentaron las tensiones de la Guerra Fría entre los Estados Unidos de América y la Unión Soviética (Fondo Monetario Internacional, 2001); la existencia del Virus de Inmunodeficiencia Humana - VIH se hizo pública por primera vez en junio de 1981 y se presentó ante el mundo como una epidemia de enormes proporciones (Herrera, s.f.), y la OMS publicó en 1980 la Clasificación Internacional de Deficiencias, Discapacidades y $\mathrm{Mi}$ nusvalías (CIDDM), la cual se puso a prueba en los años posteriores (OMS, 2001).

En Latinoamérica continuaron las desapariciones forzadas y se intensificó el terrorismo (Molina, 1988). En 1980, en Nicaragua el ejército sandinista llevó a cabo un golpe militar a la familia Somoza que instaura el Gobierno de Reconstrucción Nacional; este proceso dejó consigo una economía de guerra y cuantiosos daños humanos y económicos (Cuellar, 1999, 2000). En el mismo año, inició en El Salvador una guerra civil que duró 12 años, esta generó una amplia migración de su población hacia los Estados Unidos de América, acompañada de protestas que reclamaban dar fin al apoyo militar de ese país a El Salvador (De Cesare, 2011).

En Colombia los bajos precios del café coincidieron con una profunda crisis económica y social que se acompañó de desempleo, pobreza, hambre y enfermedad (Cano, Vallejo, Caidedo, Amador, \& Tique, 2012). Las llamadas mafias del narcotráfico comenzaron a mostrar su influencia en la vida polí- 
tica nacional, así lo reveló el triunfo de Pablo Escobar con un escaño en el Senado en las elecciones de 1982, bajo el padrinazgo de Alberto Santofimio Botero (Arias, 1993). El gobierno del presidente Virgilio Barco Vargas (1986-1990) fracasó en sus negociaciones con las FARC, al tiempo que aumentaba la violencia por cuenta de la guerra declarada por el Cartel de Medellín (Franco, 2012).

Por otro lado, el país se vio afectado por desastres de gran magnitud durante el periodo presidencial de Belisario Betancurt (1982-1986). El 31 de marzo de 1983 un sismo de 5.5 grados en la escala de Richter sacudió el departamento del Cauca y destruyó la antigua ciudad de Popayán (Gros, 1987). En 1985 el Volcán-Nevado del Ruiz hizo erupción generando una avalancha de nieve y lodo que sepultó al municipio de Armero; cerca de 25.000 personas perdieron la vida y más de 20.000 quedaron damnificadas o heridas por este hecho (Rueda, 1999). Adicionalmente, apenas unos días antes de la tragedia de Armero, el 6 de noviembre de 1985, el M-19 se tomó el Palacio de Justicia en Bogotá (Correa, 2005).

En el ámbito educativo, en enero de 1980 el entonces presidente Julio Cesar Turbay Ayala firmó el Decreto 80, con el cual se organizó el sistema de educación post-secundaria en el país y se reglamentaron las características de los programas profesionales. En su artículo 30, dicho Decreto estableció: "la formación universitaria se caracteriza por su amplio contenido social y humanitario y por su énfasis en la fundación científica e investigativa
(...) Esta modalidad se orienta en dos direcciones: hacia las disciplinas primordialmente académicas y hacia las profesiones liberales" (Ministerio de Educación Nacional, 1980).

Fue más adelante, el 25 de octubre de 1982, cuando se sancionó la primera ley de Terapia Ocupacional en el país, la Ley 31 de 1982. En ella se definía la Terapia Ocupacional como "una modalidad sistematizada de prevención, tratamiento y rehabilitación de algunas enfermedades físicas, mentales o sociales" (Art. 1) (Ministerio de Educación Nacional, 1982), destacándose el énfasis biomédico que para entonces tenía. La ley además reglamentaba las modalidades de formación en Terapia Ocupacional de la época en el sistema de educación superior: intermedia profesional, tecnológica, universitaria y avanzada de posgrado.

No ajena a la situación del país, y aunque ya con la malla que separaba el campus de la sede Bogotá del resto de la ciudad, la Universidad Nacional de Colombia era durante los ochenta centro de debates, proposiciones y luchas en defensa de lo público. Como consecuencia, fue cerrada el 16 de mayo de 1984 por espacio de un año, para reabrirse en abril de 1985 con dinámicas y estructuras distintas, "las paredes eran totalmente blancas, la cafetería, un polideportivo y las residencias sin funcionamiento, ahora la Rectoría estaba bajo el cargo de Marco Palacios, primer encargado de ejecutar reformas académicas, administrativas y estructurales" (Castro, 2009).

De otro lado, respondiendo al marco normativo trazado por la Ley 80 de 
1980, la Universidad ubicó en la modalidad de formación profesional a varias de las carreras que ofrecía, entre ellas a Terapia Ocupacional, a través del Acuerdo 156 de 1980 del Consejo Superior Universitario.

Entre tanto, las incalculables pérdidas que dejó el desastre de Armero abrieron a su vez un nuevo campo de acción para Terapia Ocupacional. Desde la Universidad Nacional de Colombia se envió una comisión a prestar diferentes servicios al eje comunitario "haciéndose evidente la necesidad de darle atención prioritaria, no solo a los "discapacitados" sino también a las personas que se vieron afectadas por el cambio sustancial en sus vidas, involucrando sus ocupaciones. Margarita González relata que los estudiantes de Terapia Ocupacional de la Escuela Colombiana de Rehabilitación también acudieron a Armero para prestar apoyo durante la crisis generada por la avalancha $(M$. González, comunicación personal, 20 de Marzo de 2015).

\section{La reforma curricular de los ochenta}

En 1988 se llevó a cabo una restructuración del programa curricular de Terapia Ocupacional de la Universidad Nacional de Colombia, la cual se hizo efectiva con la Resolución 067 de 1988, emitida por el Consejo Directivo de la Facultad de Medicina. En ella se establecieron nueve semestres académicos que implicaron una reorganización de las asignaturas teóricas, los programas académicos de campo y el trabajo de grado, y se incluyeron en los primeros semestres asignaturas como Física, Morfofisiología, Antropología, Psicología, Incapacidades Físicas y Actividades Terapéuticas, entre otras.

De acuerdo con Zapata (1994), las transformaciones vividas al final de esta década, como el enfoque social de la salud y la necesidad de los terapeutas ocupacionales del país de generar una mayor identidad profesional desde teorías propias, llevaron a que se diera este proceso de reestructuración curricular.

\section{Discusión}

La historia es definida por la Real Academia de la Lengua Española (RAE, 2012) como la narración de sucesos ocurridos en tiempo atrás que permiten la descripción y explicación de hechos pasados, que contribuyeron en el presente; sin embargo Quiceno propone que para "comprender la historia y lo que esta es, hay que mirarla desde un punto de vista distinto, apartarnos de ella y dejar que ella hable" (H. Quiceno, comunicación personal, 17 de octubre de 2014) ${ }^{8}$.

Quinceno soporta su postura en la obra de Michael Foucault (1979) ${ }^{9}$, quien argumentaba que la historia es ver más allá de los "monumentos", es

\footnotetext{
${ }^{8}$ El profesor Humberto Quiceno ha acompañado el Grupo HiTOs y al macroproyecto desde el segundo encuentro presencial del Seminario Permanente en la ciudad de Cali, realizado en octubre de 2014. Para el cuarto encuentro presencial realizado en Bogotá aportó una conferencia virtual centrada en la lectura de registros. Sus aportes han sido valiosos para la comprensión de la perspectiva histórica de Foucault.

${ }^{9}$ De igual manera, la lectura de los textos de Foucault ha sido acompañada por las investigadoras del Grupo HiTOs.
} 
el conocimiento de todo lo que hay alrededor de estos, encontrar y conocer porqué se configuró ese monumento para poderlo transformar en discursos.

Sin embargo, ante estas definiciones que ayudaron a contextualizar la investigación, la historia fue comprendida no solo como un ejercicio de identificación de sucesos del pasado, sino de re-creación de las circunstancias de un hito histórico para comprenderlo y, de esta manera, aproximarse a la reconstrucción histórica, lo que Foucault (1979) plantea como "discursos", es decir, narrativas orales u escritas que emergen a partir del conocimiento $y$ las experiencias.

Con estas conceptualizaciones como referentes, la investigación realizó no solo una lectura documental, también contó con la narrativa de la memoria viva de las personas que contribuyeron a la construcción histórica de la Terapia Ocupacional. Todo ello permitió una lectura por categorías y adicionalmente aportó la contextualización de eventos en esferas sistémicas a nivel sociopolítico, económico y cultural. Así, se pudieron identificar hitos históricos, pero también se proporciona una descripción detallada y fidedigna del devenir del programa de Terapia Ocupacional de la Universidad Nacional de Colombia desde su creación hasta los años ochenta. Ésta descripción de la historia de las tres primeras décadas del programa se considera parte de la respuesta al reto que la profesora Alicia Trujillo propuso en el 2002, en el sentido de estudiar los inicios de la profesión y articular los contextos políticos, económicos, sociales y culturales que influyeron en el surgimiento del programa y su desarrollo. Los hechos develados ayudan a comprender lo que es hoy Terapia Ocupacional en la Universidad Nacional y en el país, en palabras de una de sus protagonistas "...constituirnos como profesionales fue el resultado de las grandes luchas que hicimos y trabajamos" (A.Trujillo, Comunicación personal, 21 de abril de 2015).

\section{Conclusiones}

El trabajo realizado permitió reconocer varios hitos históricos que antecedieron el surgimiento del programa de Terapia Ocupacional en la Universidad Nacional de Colombia, entre ellos, el primera sufragio femenino, la reforma educativa que permitiría el incremento de la participación de la mujer en el ámbito universitario y la importante etapa de violencia que incrementó la demanda de servicios de rehabilitación en el país y generó la necesidad de vincular personal capacitado para ellos.

Así mismo, evidenció que durante los primeros treinta años las terapeutas ocupacionales llevaron la profesión a otros niveles, transformando sus prácticas y discursos en sintonía con las situaciones contextuales de cada época. El quehacer profesional pasó del ámbito exclusivo de la rehabilitación física y mental a la rehabilitación profesional, la promoción de habilidades en escuelas y la integración de personas en sus comunidades.

Este proyecto se transforma ahora en una invitación a la comunidad de terapeutas ocupacionales, profesionales y en formación, a creer en la historia, 
a motivarse por su estudio y encontrar nuevas brechas que permitan pensar desde lo colectivo y para la agremiación. Queda además un nuevo reto para cada sujeto de la Terapia Ocupacional: conservar de manera especial los discursos, la memoria escrita y fotográfica de las experiencias, porque cada momento es una historia que merece ser contada y escrita.

\section{Agradecimientos}

Este trabajo fue realizado con la tutoría de Aleida Fernández Moreno, apoyado y acompañado por las profesoras Jaqueline Cruz Perdomo, Clara Duarte Cuervo y Solángel García Ruiz; con quienes compartimos historias inéditas que resignificaron no solo nuestra comprensión de la historia de la Terapia Ocupacional, también la nuestra. A ellas nuestra gratitud por apreciar nuestro trabajo, por enseñarnos a desaprender y aprehender sobre el poder transformador de la historia.

\section{Referencias}

Anderson, P. (1988). Democracia y dictadura en América Latina en la década del 70. Cuadernos de Sociología, 2, 33-45.

Arias, R. (1993). La jerarquía eclesiástica colombiana y el proceso de paz del presidente Belisario Betancurt (1982-1986). Historia crítica, (8) 4, 52-65.

Cano, C., Vallejo, C., Caidedo, E., Amador, J., \& Tique, E. (2012). El mercado mundial del café y su impacto en Colombia. Borradores de Economía, 710, Recuperado de http://www.banrep.gov.co/es/ borrador-710

Capó, J. (s.f.). Escola Universitària de Relacions Laborals. Obtenido de Universitat de les Illes Balears: http://web.archive. org/save/_embed/http://www.uib.cat/ depart/deaweb/webpersonal/javiercapo/ rel_lab/temas/tema1_javi.PDF

Carrillo, D. (2014) Construyendo las ciencias sociales en Colombia: política, comunidades científicas y fundaciones filantrópicas en la Universidad Nacional de Colombia (1958-1970). Bogotá, Colombia: Universidad Nacional de Colombia.

Castro, F. (2009). Universidad Nacional, tiempos turbulentos. Obtenido de desdeabajo: https://www.desdeabajo.info/ ediciones/item/4521-universidad-nacional-tiempos-turbulentos.html

Cohen, L. (2001). Colombianas a la vanguardia. Medellín: Universidad de Antioquia.

Consejo Directivo de la Facultad de Medicina. (1988). Resolución 67 de 1988. Por la cual se aprueban unos cambios menores en el curriculum de la carrera de terapia ocupacional. Bogotá, Colombia: Universidad Nacional de Colombia.

Consejo Superior Universitario. (1966). Acuerdo 4 de 1966. Por el cual se crean las carreras de Terapia Física, Terapia Ocupacional y Terapia del Lenguaje de la Facultad de Ciencias de la Salud. Bogotá, Colombia: Universidad Nacional de Colombia.

Consejo Superior Universitario. (1976). Acuerdo 15 de 1976. Por el cual se dictan disposiciones sobre el cambio de título de las Carreras de Terapia - Facultad de Medicina-. Bogotá, Colombia: Universidad Nacional de Colombia.

Consejo Superior Universitario. (1977). Acuerdo 0016 de 1977. Por el cual se aprueba el plan de estudios para la carrera de terapia ocupacional de la facultad de medicina. Bogotá, Colombia: Universidad Nacional de Colombia.

Consejo Superior Universitario. (1977). Acuerdo 83 de 1977. Reglamenta los cargos de Directores Académicos de Carrera y Comités asesores en la Universidad Nacional. Bogotá, Colombia: Universidad Nacional de Colombia. 
Consejo Superior Universitario - CSU. (1978). Acuerdo No 12 de 1978 (Acta 6). Por el cual se deroga el Articulo 2 del Acuerdo 4 de 1966. Bogotá: Archivo Histórico y Central de la Universidad Nacional de Colombia.

Correa, H. (2005). El palacio de justicia ¿Con las armas al poder? Antecedentes, la toma, los desaparecidos. Bogotá: Carrera 7a.

Cuellar, P. (1999). De la reinserción al reencuentro. Una mirada desde la Terapia Ocupacional (Tesis Terapia Ocupacional). Bogotá: Universidad Nacional de Colombia.

Cuellar, P. (2000). Una propuesta de atención sociolaboral para exguerrilleros. Revista Ocupación Humana, 8 (3), 24-32.

De Cesare, D. (2011). 1980-1992: Guerra Civil en El Salvador. Destinys ChildrenORG. Recuperado de http://www.destinyschildren.org/es/timeline/the-salvadoran-civil-war/

Dunbar, B. (de 2008, 16 de enero). The First Person on the Moon. NASA. Recuperado de http://www.nasa.gov/audience/ forstudents/k-4/stories/first-person-onmoon.html

Fernández, A., Cruz, J., Duarte, C., \& García, S. (2015, 27 de febrero). Cincuenta años de Terapia Ocupacional en Colombia. San Juan de Pasto.

Fondo Monetario Internacional. (2001, noviembre). La liberalización del comercio mundial y los países en desarrollo. Fondo Monetario Internacional. Recuperado de http://www.imf.org/external/np/exr/ $\mathrm{ib} / 2001 / \mathrm{es} / 110801 \mathrm{~s} . \mathrm{htm}$

Foucault, M. (1979). La arqueología del saber. España: Siglo veintiuno Editores.

Franco, C. (2012, 18 de febrero). La verificación en un eventual proceso de paz. Recuperado de https://www.files.ethz. ch/isn/151973/wp-10.pdf

Gómez, C., Munar, C., Parrado, Y., Urbano, G., Rodríguez, M., Vargas, E., \& Velan- dia, A. (2011). Tres escuelas una historia: formación de enfermeras en la Universidad Nacional de Colombia 1920-1957. Bogotá: Universidad Nacional de Colombia.

Gutiérrez, R. (Compositor). (2015). Inauguración Catedra de Sede José Félix Patiño. Bogotá.

Gros, C. (Marzo de 1987). Popayán dos años después: Autopsia de un desastre. Recuperado de Universidad del Valle: http:// cms.univalle.edu.co/socioeconomia/media/ckfinder/files/Popayan\%20dos\%20 anos $\% 20$ despues $\% 20$ Autopsia $\% 20$ de\%20un\%20desastre.pdf

Grupo de Investigación Historias de las Terapias Ocupacionales -HiTOs. (2014). Boletín de prensa No. 1. Alrededor de 50 terapeutas ocupacionales se reunieron para hacer historia. Universidad del VaIle, Cali, Colombia.

Grupo de Investigación Historias de las Terapias Ocupacionales -HiTOs. (2015) Boletín informativo No. 2. Bogotá, Colombia.

Harina, D. (2010, octubre). La Gira de Fidel Castro por América Abril - Mayo de 1959. Fluctuaciones e Implicancias Político Ideológicas. Universidad de Palermo. Recuperado de http://dspace.palermo. edu/dspace/bitstream/10226/1188/1/ Harina,\%20Daniela.pdf

Herrera, C. (s.f.). Sida, ahora una enfermedad controlable. Obtenido de Agencia de Noticias UN: http://historico.unperiodico.unal.edu.co/ediciones/109/09. html

Hobsbawn, E. (2000). Historia del siglo XX. 1914-1991. Crítica Barcelona. Recuperado de http://www.historiasiglo20.org/ GF/

Instituto Materno Infantil. (2003). Programa Materno Infantil. Amor, Calor y Leche Materna. Recuperado de http://madrecanguro.tripod.com/index1024.htm

Jaimes, G. E. (2012). M-19 - Universidad Nacional de Colombia. Recupe- 
rado de http://www.bdigital.unal.edu. co/9917/1/468440.2012.pdf

Laserna, R. (1995, marzo). Programa para el desarrollo del área de orientación ocupacional laboral. Bogotá, Colombia: Universidad Nacional de Colombia.

López, R. (2002). La Univerdidad femenina, las ideologías de género y el acceso de las colombianas a la educación superior 1940 - 1958. Historia de la educación Latinoamericana , 67-90.

Mengo, R. (2004). Serie Tesis Doctorales. Incidencia de los golpes de Estado de los 60 en la prensa de Córdoba (República Argentina). Argentina: Universidad de La Laguna.

Ministerio de Educación Gobierno de Chile. (2014). Biblioteca Nacional Digital de Chile. Recuperado de http://www.memoriachilena.cl/602/w3-article-31395. html

Ministerio de Educación Nacional. (1980). Decreto número 80 de 1980. Por el cual se organiza el sistema de educación post-secundaria. Recuperado de http:// www.mineducacion.gov.co/1621/articles-102556_archivo_pdf.pdf

Ministerio de Educación Nacional. (1982). Ley 31 de 1982. Por la cual se regula la profesión de Terapia Ocupacional y se dictan otras disposiciones. Recuperado de http://www.mineducacion.gov. co/1621/articles-103938_archivo_pdf. pdf

Molina, A. L. (1988). La desaparición forzada de personas en América Latina. Recuperado de http://www.desaparecidos. org/nuncamas/web/investig/biblio_theissen_01.htm

Mouffe, C. (1999). El retorno de lo político. Barcelona: Paidós.

Organización Mundial de la Salud -OMS. (2001,9 de abril). Clasificación internacional del funcionamiento, de la discapacidad y de la salud. Informe de la Secretaría. Recuperado de http://apps.
who.int/gb/archive/pdf_files/WHA54/ sa5418.pdf

Pardo, J. (1972). Curso para la Formación de Médicos Especialistas en Rehabilitación. Bogotá.

Pardo, J. (s.f). Carreras de Terapia. Bogotá: Facultad de Medicina. Universidad Nacional de Colombia.

Peñas, O. L. (2006). Terapia ocupacional en Colombia: cuatro décadas de posicionamiento y servicios en la sociedad. Revista Facultad de Medicina, 54(4), 229-231.

RAE, (2012). Real Academia Española. Obtenido de Diccionario de la lengua española. Recuperado de http://lema.rae.es/ drae/?val=HISTORIA

Registro de personal docente. (s.f.). Historia laboral. Patricia Ann de Pardo. Bogotá, Colombia: Archivo Central e Histórico. Universidad Nacional de Colombia.

Reyes, N. (2011). Necesidades de hoy: memoria histórica, patrimonio, costumbres y/o tradiciones e identidad cultural en la familia. Recuperado de Contribuciones a las Ciencias Sociales: http://www.eumed.net/rev/cccss/13/ncrp2.html

Rueda, J. (1999, septiembre). La avalancha de Armero: noviembre 13 de 1985. Banco de la República. Obtenido de http:// www.banrepcultural.org/node/32977

Tribuna Roja. (1977, 29 de octubre). El Paro Cívico Nacional, imperó la ley de los de abajo. Recuperado de http://moir.org. co/web/el-paro-civico-nacional-imperola-ley-de-los-de-abajo/

Trujillo, A. (1981). Estudios sobre la Estructura Orgánica de las Carreras de Terapia Física, Terapia Ocupacional y Terapia de Lenguaje en la Universidad Nacional. Bogotá: Universidad Nacional de Colombia.

Trujillo, A. (2002). Terapia Ocupacional. Conocimiento y Práctica en Colombia. Bogotá: Universidad Nacional. 
Trujillo, A., Torres, M., Méndez, J., \& Carrizosa, L. (2011). Terapia Ocupacional Conocimiento y Compromiso Social. Bogotá: Universidad Nacional de Colombia.

UNESCO. (1998). Mujeres y Educación Superior: Cuestiones y Perspectivas. Recuperado de http://unesdoc.unesco.org/ images/0011/001136/113684so.pdf

Urrego, M. (2002). Intelectuales, Estado y nación en Colombia: de la guerra de los mil días a la Constitución de 1991. Bogotá: Universidad Central-CIUD, Siglo del Hombre Editores.

Zapata, M. V. (1994). Orientación en la formación universitaria de Terapia Ocupacional en Colombia. En Terapia Ocupacional y universidad (p.p.11-18). Bogotá: Universidad Nacional de Colombia. 